\begin{tabular}{|c|c|c|c|c|}
\hline $\begin{array}{l}\text { F O U N D A T I O N S } \\
\text { Vol. } 42\end{array}$ & $\mathrm{OF}$ & $\underset{(2017)}{\operatorname{C~O} M \text { P U T I N G A D }}$ & D E C I S I O N & $\begin{array}{r}\text { S C I E N C E S } \\
\text { No. } 1\end{array}$ \\
\hline $\begin{array}{ll}\text { DE } & \text { DE GRUYTER } \\
\text { OPEN } & \end{array}$ & & DOI: $10.1515 /$ fcds-2017-0002 & & $\begin{array}{r}\text { ISSN } 0867-6356 \\
\text { e-ISSN 2300-3405 }\end{array}$ \\
\hline
\end{tabular}

\title{
VELOCITY CONTROLLER FOR A CLASS OF VEHICLES
}

\author{
Przemyslaw HERMAN, Wojciech ADAMSKI *
}

\begin{abstract}
This paper addresses the problem of velocity tracking control for various fully-actuated robotic vehicles. The presented method, which is based on transformation of equations of motion allows one to use, in the control gain matrix, the dynamical couplings existing in the system. Consequently, the dynamics of the vehicle is incorporated into the control process what leads to fast velocity error convergence. The stability of the system under the controller is derived based on Lyapunov argument. Moreover, the robustness of the proposed controller is shown too. The general approach is valid for 6 DOF models as well as other reduced models of vehicles. Simulation results on a $6 \mathrm{DOF}$ indoor airship validate the described velocity tracking methodology.
\end{abstract}

Keywords: Marine vehicle, hovercraft, indoor airship, velocity transformation, nonlinear control.

\section{Introduction}

Autonomous and remotely operated vehicles are becoming a key component in various aspects of environment research. To the group of systems belong ships, hovercrafts, submarines, airships, and others. Accurate trajectory tracking control is one of crucial problems concerning these vehicles.

A sliding mode control based on the velocity tracking idea for unmanned surface vessel systems was described in [2]. As it arises from [5] the velocity control system (for guided motion control system) based on velocity tracking is useful for marine vehicles. Surge and yaw relative velocity control of ships can be also based on velocity tracking idea [6]. Some velocity controllers for an autonomous underwater vehicle were proposed in [8]. Tracking control laws for marine vehicles (underwater vehicles or surface ships) can be found, e.g. in [10]. Moreover, the tracking control of aerial

* Chair of Control and Systems Engineering Poznan University of Technology ul. Piotrowo 3a, 60-965 Poznan, Poland, \{przemyslaw.herman, wojciech.adamski\}@put.poznan.pl 
indoor blimp robots is sometimes realized utilizing velocity based simple (not global) controller [11]. The velocity controllers are also a part of the control system for an AUV with nonlinear dynamics. Velocity control algorithms are applied for other systems, for example quad-rotors [22] or unmanned helicopters [24]. The algorithms are useful for reduction of the velocity error between the desired camera velocity and the actual velocities in the camera frame [22] or control of steady-state force [24]. In practical applications the velocity control can be realized by PI controller from gain tuning formulas based on the identified parameters [30].

The problem of the velocity tracking control for a class of fully actuated vehicles in the body-fixed frame is addressed in this paper. Fully actuated vehicles are often considered in the literature, e.g. in for underwater vehicle [9, 12], surface vehicles [26, $28]$, hovercrafts $[13,25]$ or airships [20, 29]. The main difference between our velocity tracking controller and the aforementioned approaches relies on that we include the vehicle dynamics directly into the velocity control gain matrix. Thus, the strategy is also different from those solutions because of the different idea of control, namely taking into the control gain matrix the dynamics couplings of the vehicle. Thanks this property the matrix is strictly related to the moving system. Comparing with previous studies concerning underwater vehicles, some surface vehicles and indoor airships this paper offers different approach to control algorithms. In classical algorithms the elements of the control gain matrix are selected without taking into account dynamical and geometrical parameters of the system. The main contribution of this paper is a velocity tracking control algorithm and its application for an airship model. Novelty of the presented approach relies on that the control algorithm uses transformation of the velocity vector based on an inertia matrix decomposition method. Moreover, each new rate (speed) is regulated separately in the sense that dynamical couplings of the system are included in it. Consequently, the response of the system is fast (and always depends on the couplings in the system), what means that the velocity error decreases quickly. Therefore, the desired trajectory can be reached in short time. Another benefit arising from the use of the controller relies on that the velocity gain matrix contains parameters of the system and strictly depends on its dynamics (for various vehicles the gain coefficients can be quite different). The proposed, in this work, velocity control algorithm is new. The considered in [16] PD controller was based on the same equations of motion but its idea was quite different because the tracking problem was absent. Besides, the obtained controller is universal in the sense that: 1) it can be applied both for 6 DOF as well as for vehicles moving in a horizontal or vertical plane, 2) it is appropriate for various system, namely fully actuated marine vehicles, hovercrafts, and indoor airships. The stability of the system under the proposed controller using the Lyapunov direct method whereas robustness to parameter changes is shown in simulation experiment.

The remainder of this paper is organized as follows. In Section 2, the mathematical model describing the class of vehicles is introduced. The proposed velocity tracking controller is presented in Section 3. Simulation results for an indoor airship model are shown in Section 4. Section 5 consists of concluding remarks about the results and future research. 


\section{Transformed equations of motion}

Taking into account the inertial generalized forces, the hydrodynamic effects, the gravity, and buoyancy contributions as well as the effects of the actuators (thrusters), the dynamic model of an underwater vehicle (Figure 1) using the notation proposed by $[10]$ can be written in the matrix form as:

$$
\begin{aligned}
& M \dot{\nu}+C(\nu) \nu+D(\nu) \nu+g(\eta)=\tau, \\
& \dot{\eta}=J(\eta) \nu .
\end{aligned}
$$

The equations of motion can be also used for airships under assumptions that they moves slowly and the environmental factors are weak. For example the equations are appropriate for indoor flight of an airship. In (1) and (2) $\nu=[u, v, w, p, q, r]^{T}$, $\eta=[x, y, z, \phi, \theta, \psi]^{T}$ are vectors of velocities (in the body-fixed frame) and position and Euler angles (in the earth-fixed frame), respectively. Moreover, $J(\eta) \in R^{6 \times 6}$ is the Jacobian transformation matrix mapping the body-fixed frame to the earth-fixed frame. The matrix $M \in R^{6 \times 6}$ denotes the inertia matrix (including the rigid body inertia matrix and the added mass matrix), and satisfies $M=M^{T}>0$, and $\dot{M}=0$, whereas the matrix $C(\nu) \in R^{6 \times 6}$ denotes the Coriolis-centripetal matrix due to the rigid body and the added mass (that satisfies $C(\nu)=-C^{T}(\nu)$ ). Moreover, the matrix $D(\nu)>0 \in R^{6 \times 6}, \forall \nu \in R^{6}, \nu \neq 0$ denotes the damping, while the vector $g(\eta) \in R^{6}$ is the a vector of gravitational and buoyancy forces, and $\tau \in R^{6}$ is the vector of control inputs influencing the vehicle.

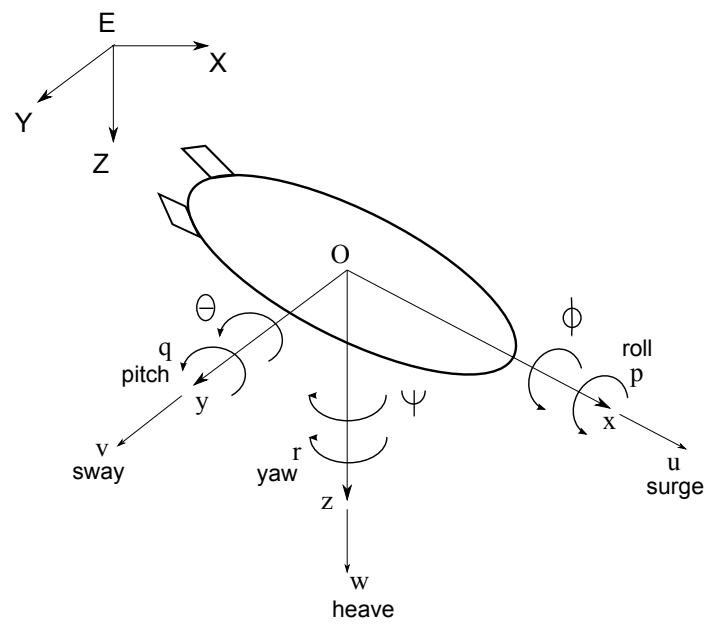

Figure 1. The coordinate system for $6 \mathrm{DOF}$ vehicle.

Remark 1. For the assumed vehicle model the inertia matrix $M$ is constant, symmetric, and in general, non-diagonal, i.e. it contains off diagonal elements. As it arises from the literature, e.g. [10] for a class of vehicle models such approximation is allowable. The matrix $M$ can be approximated as a symmetric matrix for vessels 
moving with low speed. This condition is fulfilled, for example, for indoor airship flight because of weak environmental disturbances. For outdoor flying airships the environmental effects are great and components of the inertia matrix as well as the added mass coefficients depend strongly on geometry, fluid flow rates and other uncertainties. Similarly, if wind will be taken into account then the equations (1) and (2) will be inadequate and the proposed control algorithm is insufficient. If the external disturbances are great (for outdoor airship) then in the Eq.(1) the term defining their model must be added and consequently the controller have to be built in different way.

Remark 2. The equations (1) and (2) can be applied for ocean or marine vehicles as well as for airships during indoor experiments. Such examples can be found: (1) for underwater vehicles in $[12,17],(2)$ for surface vessels in $[7,18]$ or hovercrafts in $[13,21]$, (3) for indoor airships in [23, 31].

The control strategy is based on transformed equations of motion with the identity inertia matrix. Thus, we must decompose the inertia matrix $M$ into two matrices:

$$
M=\Phi^{T} \Phi
$$

and next define some new rates:

$$
\zeta=\Phi \nu
$$

According to Remark 1 and the assumed decomposition method the matrix $\Phi$ in (4) is upper triangular, invertible and have constant elements only. In such case the time derivative of $\zeta$ is $\dot{\zeta}=\Phi \dot{\nu}$.

There are various decomposition methods of the matrix $M$. In this paper, we refer to the method described in [14] which is based on generalized velocity components introduced in [19] and applied for robotics manipulators in [15]. The decomposition strategy is easy comprehensible and convenient for numerical implementation. However, using any other method the general result will be similar but not necessary the same.

Recalling [16] we insert (3) and $\nu$ obtained from (4) into (1), and pre-multiply both sides by $\Phi^{-T}$ (i.e. $\left(\Phi^{T}\right)^{-1}$ ) we get the transformed equations of motion as follows:

$$
\begin{aligned}
& \dot{\zeta}+C_{\zeta}(\zeta) \zeta+D_{\zeta}(\zeta) \zeta+g_{\zeta}(\eta)=\varpi, \\
& \dot{\eta}=J(\eta) \Phi^{-1} \zeta
\end{aligned}
$$

where the matrices and the vectors are:

$$
\begin{aligned}
& C_{\zeta}(\zeta)=\Phi^{-T} C(\nu) \Phi^{-1}, \\
& D_{\zeta}(\zeta)=\Phi^{-T} D(\nu) \Phi^{-1}, \\
& g_{\zeta}(\eta)=\Phi^{-T} g(\eta), \\
& \varpi=\Phi^{-T} \tau .
\end{aligned}
$$

Equations (5) and (4) together with (6) describe the motion of the vehicle in terms of the transformed rates. The dynamical coupling between components of the vector $\nu$ are included in the vector $\zeta$ as well as in other terms of (5). This fact allows one to observe some phenomena arising from the dynamical coupling which are not easy noticeable for (1) and (2). 


\section{$3 \quad$ Velocity tracking control algorithm}

The control objective is to design a velocity trajectory tracking control algorithm in the body-fixed frame representation. What is important, in the proposed algorithm the dynamical coupling in the vehicle are included in the velocity gain matrix. Thanks this property the gain matrix is strictly related to the investigated moving system. Novelty of the approach arises thus from the main idea, i.e. the use of dynamical couplings existing in the vehicle for fast vehicle velocity error convergence.

\subsection{Nonlinear controller}

The controller decoupled in the sense of the vector of the transformed variables $\zeta$ is presented in the proposition given below.

Theorem 1. Consider the vehicle dynamic model (5) and (4) together with the following controller:

$$
\varpi=\dot{\zeta}_{r}+C_{\zeta}(\zeta) \zeta_{r}+D_{\zeta}(\zeta) \zeta_{r}+g_{\zeta}(\eta)+k_{D} s_{\zeta}+\Phi^{-T} k_{I} z
$$

where

$$
\begin{aligned}
& z=\int_{0}^{t_{k}} \tilde{\nu}(t) d t, \\
& \zeta_{r}=\Phi\left(\nu_{d}+\Lambda z\right), \\
& s_{\zeta}=\zeta_{r}-\zeta=\Phi(\tilde{\nu}+\Lambda z), \\
& \dot{s}_{\zeta}=\dot{\zeta}_{r}-\dot{\zeta}=\Phi(\dot{\tilde{\nu}}+\Lambda \tilde{\nu}),
\end{aligned}
$$

and $\tilde{\nu}=\nu_{d}-\nu$ is the velocity error vector, $k_{D}=k_{D}^{T}>0, k_{I}=k_{I}^{T}>0$, and $\Lambda=\Lambda^{T}>0$, and $t$ means the time. The equilibrium point $\left[s_{\zeta}^{T}, z^{T}\right]^{T}=0$ is globally exponentially stable.

Remark 3. For simplicity we will assume that $k_{D}, k_{I}$, and $\Lambda$ are constant and diagonal. If we use the symmetric matrices, then we select $\Lambda=k_{I}^{-1} k_{D}$ (for the diagonal matrices this condition is not necessary). The integral action term is used here to improve control performance and to obtain correct reaction of the system.

Remark 4. Note that the control algorithm (11) is realized in the vehicle body-frame. The obtained results can be transformed next into the earth-frame using the kinematic relationship (6).

Proof. The closed-loop system (5) and (4) together with the controller (11) can be written as follows:

$$
\begin{aligned}
& \dot{\zeta}+C_{\zeta}(\zeta) \zeta+D_{\zeta}(\zeta) \zeta+g_{\zeta}(\eta) \\
& =\dot{\zeta}_{r}+C_{\zeta}(\zeta) \zeta_{r}+D_{\zeta}(\zeta) \zeta_{r}+g_{\zeta}(\eta)+k_{D} s_{\zeta}+\Phi^{-T} k_{I} z
\end{aligned}
$$

what leads to:

$$
\dot{s}_{\zeta}+\left[C_{\zeta}(\zeta)+D_{\zeta}(\zeta)+k_{D}\right] s_{\zeta}+\Phi^{-T} k_{I} z=0
$$


As a Lyapunov function candidate the following expression can be proposed:

$$
\mathcal{L}\left(s_{\zeta}, z\right)=\frac{1}{2} s_{\zeta}^{T} s_{\zeta}+\frac{1}{2} z^{T} k_{I} z
$$

Calculating the time derivative of the function $\mathcal{L}(18)$ one obtains:

$$
\dot{\mathcal{L}}\left(s_{\zeta}, z\right)=s_{\zeta}^{T} \dot{s}_{\zeta}+\tilde{\nu}^{T} k_{I} z
$$

Recalling the relationship (17) one gets:

$$
\dot{\mathcal{L}}\left(s_{\zeta}, z\right)=s_{\zeta}^{T}\left[-C_{\zeta}(\zeta) s_{\zeta}-D_{\zeta}(\zeta) s_{\zeta}-k_{D} s_{\zeta}-\Phi^{-T} k_{I} z\right]+\tilde{\nu}^{T} k_{I} z
$$

Recalling (7), we can write $s_{\zeta}^{T} C_{\zeta}(\zeta) s_{\zeta}=s_{\zeta}^{T} \Phi^{-T} C(\nu) \Phi^{-1} s_{\zeta}$. Denoting now $s=\Phi^{-1} s_{\zeta}$ we obtain $s_{\zeta}^{T} C_{\zeta}(\zeta) s_{\zeta}=s^{T} C(\nu) s=0$ because $s^{T} C(\nu) s=0$ for all $s \in R^{n}$ (the matrix $C(\nu)$ is a skew-symmetric one) [10]. Therefore, taking into account (14) we have:

$$
\begin{aligned}
& \dot{\mathcal{L}}\left(s_{\zeta}, z\right)=-s_{\zeta}^{T}\left[D_{\zeta}(\zeta)+k_{D}\right] s_{\zeta}-s_{\zeta}^{T} \Phi^{-T} k_{I} z+\tilde{\nu}^{T} k_{I} z \\
& =-s_{\zeta}^{T}\left[D_{\zeta}(\zeta)+k_{D}\right] s_{\zeta}-z^{T} \Lambda^{T} k_{I} z .
\end{aligned}
$$

Using (8) and denoting a quadratic matrix $k_{D}^{*}=\Phi^{-T} D(\nu) \Phi^{-1}+k_{D}$ (this matrix is positive definite, i.e $k_{D}^{*}>0$ if selection of $k_{D}$ is appropriate, namely the sum of these matrices gives a strictly positive definite matrix) the two latter terms of (21) may be bounded by:

$$
\begin{aligned}
& -s_{\zeta}^{T} k_{D}^{*} s_{\zeta} \leq-\lambda_{\min }\left\{k_{D}^{*}\right\}\left\|s_{\zeta}\right\|^{2}, \\
& -z^{T} \Lambda^{T} k_{I} z \leq-\lambda_{\min }\left\{\Lambda^{T} k_{I}\right\}\|z\|^{2} .
\end{aligned}
$$

Consequently, it also holds that:

$$
\dot{\mathcal{L}}\left(s_{\zeta}, z\right)=-\left[\begin{array}{c}
\left\|s_{\zeta}\right\| \\
\|z\|
\end{array}\right]^{T} \underbrace{\left[\begin{array}{cc}
\lambda_{\min }\left\{k_{D}^{*}\right\} & 0 \\
0 & \lambda_{\min }\left\{\Lambda^{T} k_{I}\right\}
\end{array}\right]}_{A}\left[\begin{array}{c}
\left\|s_{\zeta}\right\| \\
\|z\|
\end{array}\right] .
$$

Note that the matrix $A$ is positive definite (the matrix $\lambda_{\min }\left\{\Lambda^{T} k_{I}\right\}$ is composed of diagonal matrices or $\left.\lambda_{\min }\left\{\Lambda^{T} k_{I}\right\}=\lambda_{\min }\left\{k_{D}\right\}\right)$. Thus, assuming $\lambda_{\min }\{A\}>0\left(\lambda_{\min }\right.$ is the minimal eigenvalue of the matrix $A$ ) one can find an upper bound of the time derivative. Denoting now $x=\left[\left\|s_{\zeta}\right\|,\|z\|\right]^{T}$ one can write:

$$
\dot{\mathcal{L}}(t, x) \leq-\lambda_{\min }\{A\}\|x\|^{2},
$$

for all $t \geq 0$ and $x \in R^{2 \mathcal{N}}$.

Therefore, based on the Lyapunov direct method [27], we conclude that the state space origin of the system (5), (4) together with the controller (11):

$$
\lim _{t \rightarrow \infty}\left[\begin{array}{c}
s_{\zeta}(t) \\
z(t)
\end{array}\right]=0
$$

is globally exponentially convergent. 


\subsection{Advantages and properties of the controller}

Consider some properties and benefits resulting from the use of the proposed controller. The controller (11) is expressed in terms of the vector $\zeta$. The input signal is obtained from the relationship (10) calculating $\tau=\Phi^{T} \varpi$ (the matrix $\Phi^{T}$ is invertible and symmetric). As a result, the velocity gain matrix in the fifth term is $\Phi^{T} k_{D} \Phi$. Assuming that $k_{D}=\delta \cdot I$ ( $I$ denotes the identity matrix whereas $\delta$ is a constant value) we have the following gain matrix $K_{D}=\Phi^{T} k_{D} \Phi=\delta \cdot M$.

1. First benefits resulting from the controller is that the gain coefficients (they depend on the parameter set) are strictly related to the dynamics of the vehicle hidden in the matrix $K_{D}$. Consequently, it is not necessary to choose a symmetric gain matrix because the matrix $K_{D}$ is a symmetric one. The diagonal matrix $k_{D}$ serves rather for precise tuning of the controller.

2. The use of the gain matrix $K_{D}=\Phi^{T} k_{D} \Phi$, because including the dynamics of the vehicle, allows one to ensure the same control coefficients even if the parameters set in not known exactly.

3. The control gains are selected for the tested system that enables to avoid searching their values using experience of the researcher only.

\section{Simulation results}

In order to investigate effectiveness of the control algorithm, a computer simulation was performed for the motion of a 6 DOF model of the airship AS500 (assuming indoor flight). The vehicle parameters based on the report [4] were used also in [1].

The symbols mean: $\mathrm{L}$ is length and $\mathrm{b}$ is breadth of the vehicle. The body weight is $W=m g$, and the buoyancy force $B=\rho g \nabla$ with $\rho=1.225 \mathrm{~kg} / \mathrm{m}^{3}, g=9.81 \mathrm{~m} / \mathrm{s}^{2}$ where $\nabla=10 \mathrm{~m}^{3}$. The center of gravity is $r_{G}=\left[x_{G}, y_{G}, z_{G}\right]$ with $x_{G}=-3.6644$ $\mathrm{m}, y_{G}=0 \mathrm{~m}, z_{G}=0.4032 \mathrm{~m}$ and the center of buoyancy is $r_{B}=\left[x_{B}, y_{B}, z_{B}\right]$ with $x_{B}=y_{B}=z_{B}=0 \mathrm{~m}$. The airship parameters are given in Table 1 . The maximal possible forces and torques applied by the control system were assumed as follows: $F_{\max x, y, z}=107,13,40 \mathrm{~N}, T_{\max x, y, z}=27,267,27 \mathrm{Nm}$. These values were taken from [3] for the airship AS800 (this airship is bigger but its construction is similar to the used airship).

Two cases were investigated, namely: nominal parameters set and $50 \%$ weight reduction.

Case 1 - set of nominal parameters. In the first test the airship nominal parameters were taken into consideration. For tracking it is assumed the following desired velocity profile:

$$
\nu_{d}=[\sin (\pi / 20 \cdot t)+2, \quad 0, \quad \sin (\pi / 25 \cdot t), \quad 0, \quad 0, \quad 0.1 \cdot \cos (p i / 15 \cdot t)]^{T} .
$$


Table 1. Parameters of the airship and added masses $a_{i j} i, j=1, \ldots, 6$

$\begin{array}{llllll}\text { Symbol } & \text { Value } & \text { Unit } & \text { Symbol } & \text { Value } & \text { Unit } \\ L & 7.8 & \mathrm{~m} & a_{11} & 1.247 \rho & \mathrm{kg} \\ b & 1.9 & \mathrm{~m} & a_{15} & 0 & \mathrm{~kg} \\ m & 18.375 & \mathrm{~kg} & a_{22} & 17.219 \rho & \mathrm{kg} \\ I_{x} & 11.7473 & \mathrm{kgm}^{2} & a_{24} & -1.231 \rho & \mathrm{kg} \\ I_{y} & 268.1748 & \mathrm{kgm}^{2} & a_{26} & -56.893 \rho & \mathrm{kg} \\ I_{z} & 260.0255 & \mathrm{kgm}^{2} & a_{33} & 16.671 \rho & \mathrm{kg} \\ J_{x y} & 0 & \mathrm{kgm}^{2} & a_{35} & 55.269 \rho & \mathrm{kg} \\ J_{x z} & -21.7069 & \mathrm{kgm}^{2} & a_{44} & 13.38 \rho & \mathrm{kgm}^{2} \\ J_{y z} & 0 & \mathrm{kgm}^{2} & a_{46} & 3.658 \rho & \mathrm{kgm}^{2} \\ & & & a_{55} & 311.942 \rho & \mathrm{kgm}^{2} \\ & & & a_{66} & 316.814 \rho & \mathrm{kgm}^{2}\end{array}$

The gain coefficients of the controller (based on calculations using the genetic algorithm) were selected as follows:

$$
\begin{aligned}
& k_{D}=\operatorname{diag}\{20,20,20,80,80,80\}, \\
& k_{I}=\operatorname{diag}\{50,50,50,45,45,45\}, \\
& \Lambda=\operatorname{diag}\{0.9,0.9,0.9,0.2,0.2,0.2\} .
\end{aligned}
$$

For the classical control algorithm (namely in which the elements of the control gain matrix were selected without taking into account dynamical and geometrical parameters of the system) the gain matrices (also based on the same genetic algorithm) were assumed as:

$$
\begin{aligned}
& k_{D}=\operatorname{diag}\{159,159,159,107,107,107\}, \\
& k_{I}=\operatorname{diag}\{104,104,104,168,168,168\}, \\
& \Lambda=\operatorname{diag}\{0.03,0.03,0.03,1.83,1.83,1.83\} .
\end{aligned}
$$

The chosen sets of control gains are different because in the proposed controller the parameters of the system are taken into account, and additionally control gains were found from the genetic algorithm. In the classical controller the gains were calculated based on the genetic algorithm only. For the same reason values of $k_{D}, k_{I}, \Lambda$ are bigger for the classical controller (with exception of the first elements of $\Lambda$ ). The results obtained from both control schemes are presented in Figures 2-4. One of criterion for comparison of different algorithms is the control effort, measured by the control signals, which should have similar values.

In Figures 2(a) and 2(b) the desired linear and angular velocities are given. Three profiles are time varying according to different sinusoidal functions.

Figure 3(a) presents linear velocity error for each variable. It is observable that all linear errors tend to zero quickly (after about 10 second). Similarly, as it arises from Figure 3(b), the angular velocity errors are reduced in a short time (but more slowly than the linear velocity errors). Such fast response of the system results from the fact 


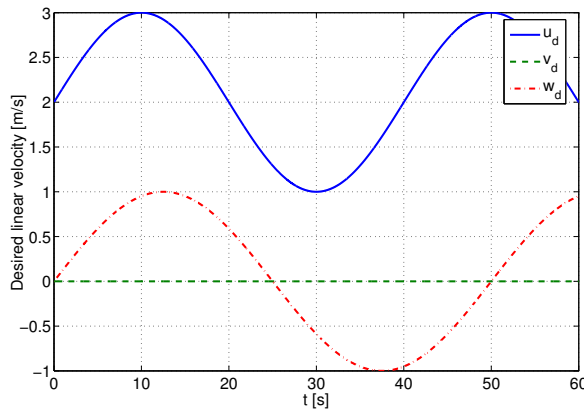

(a)

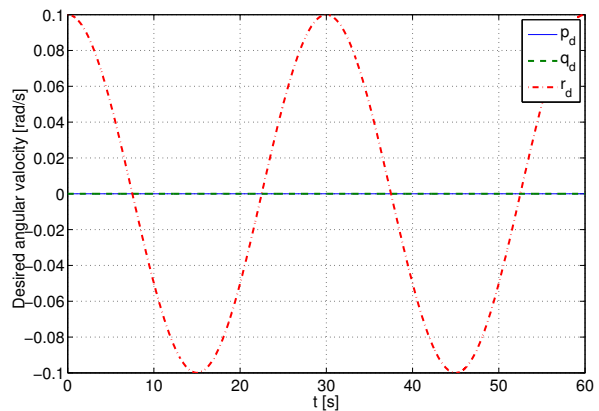

(b)

Figure 2. Case 1 and Case 2: a) desired linear velocities $u_{d}, v_{d}, w_{d}$, b) desired angular velocities $p_{d}, q_{d}, r_{d}$.

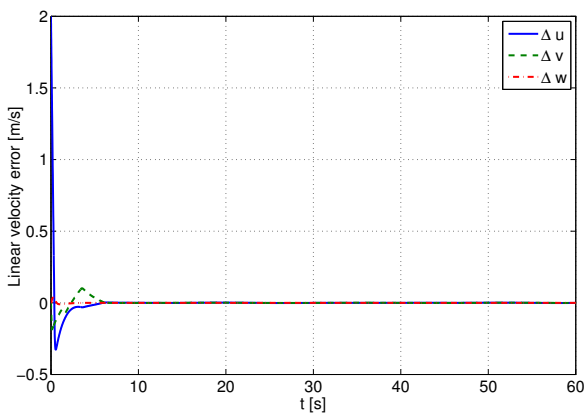

(a)

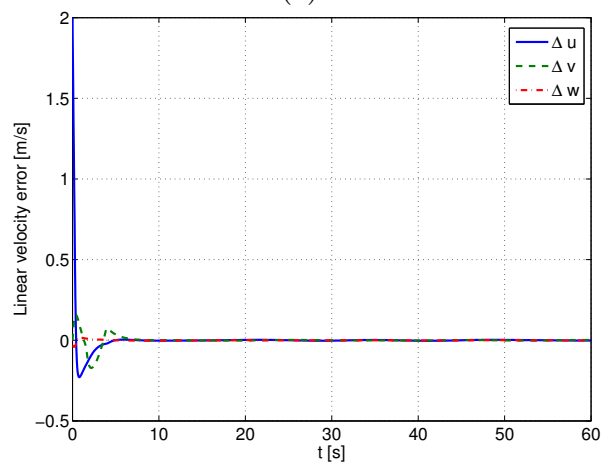

(c)

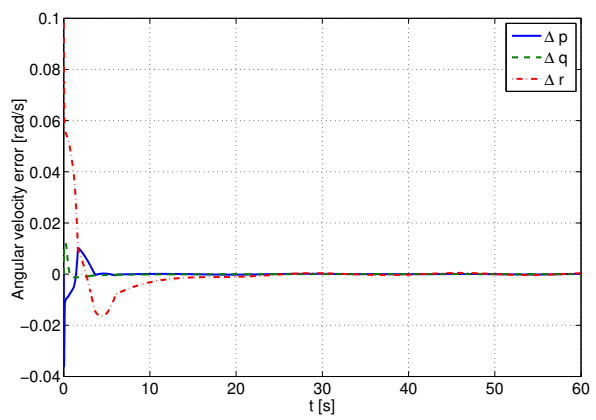

(b)

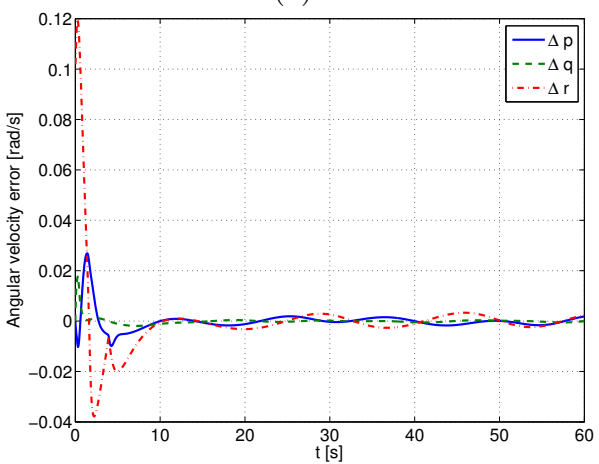

(d)

Figure 3. Case $1(\Delta \nu \equiv \tilde{\nu})$ : a) (proposed controller) linear velocity errors $\Delta u, \Delta v, \Delta w, \mathrm{~b}$ ) (proposed controller) angular velocity errors $\Delta p, \Delta q, \Delta r, \mathrm{c}$ ) (classical controller) linear velocity errors $\Delta u, \Delta v, \Delta w, \mathrm{~d}$ ) (classical controller) angular velocity errors $\Delta p, \Delta q, \Delta r$. 


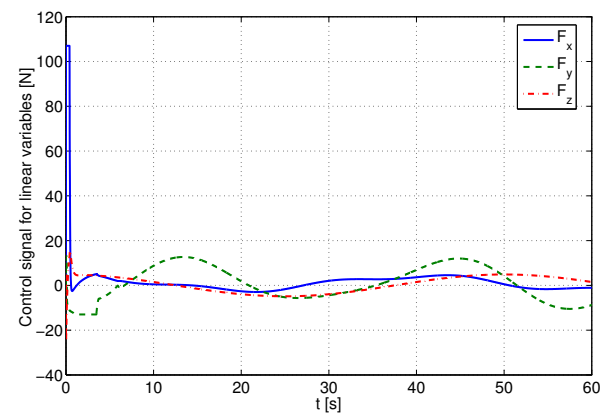

(a)

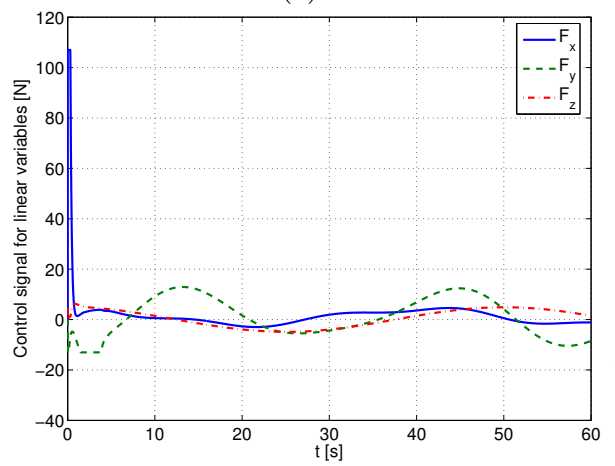

(c)

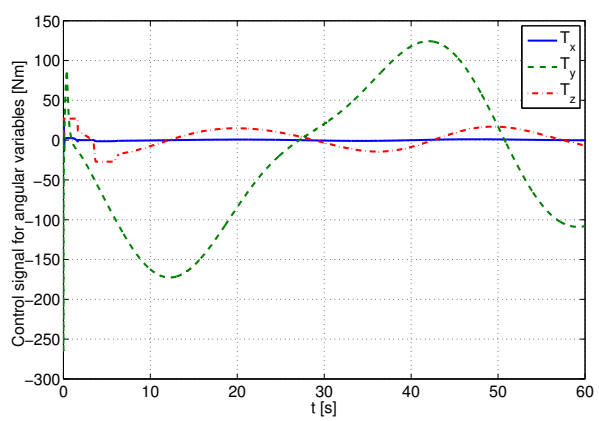

(b)

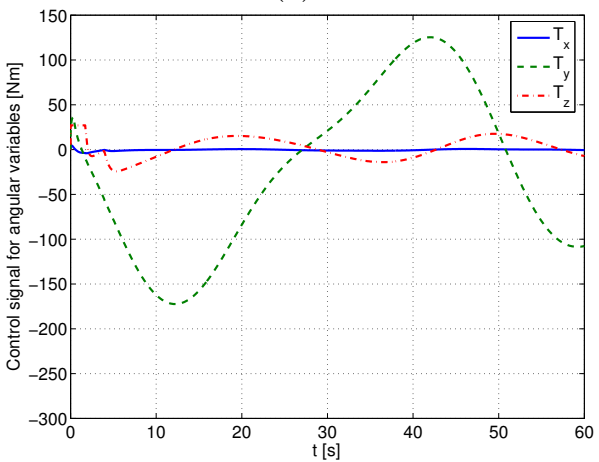

(d)

Figure 4. Case 1 - control signals: a) (proposed controller) applied forces $F_{x}, F_{y}, F_{z}$, b) applied torques (proposed controller) $T_{x}, T_{y}, T_{z}$, c) (classical controller) applied forces $F_{x}, F_{y}, F_{z}$, b) applied torques (classical controller) $T_{x}, T_{y}, T_{z}$. 
that in the control process the vehicle dynamics is included (in the velocity gains). From Figure 3(c) we observe that the classical controller gives similar linear velocity errors as the proposed one. However, the angular velocity errors (Figure 3(d)) are bigger and do not tend to zero.

The applied forces are depicted in Figure 4(a). They are quickly reduced below 20 N. Next as it arises from Figure 4(b) the applied torque $T_{y}$ has the maximal value over $100 \mathrm{Nm}$ what means that the dynamical couplings in this direction are stronger than for other directions. From the conducted test it is observable that the control goal, namely velocity error reduction, is realized in short time. It results from two reasons: directly relationship between the system dynamics and the control gain matrix because of $K_{D}=\Phi^{T} k_{D} \Phi$ and the use of the integral action term. Moreover, the applied forces and torques are related to the dynamical couplings between the velocity variables. As it arises from Figures 4(c) and 4(d) the control effort for the classical control algorithm is comparable with the effort for the proposed controller.

Case 2 (robustness test) - $50 \%$ weight reduction (both the mass and inertia were reduced). In this test sensitivity to the parameter changes of the controller was investigated. The set of controller gains as well as the desired velocity profiles were the same as in Case 1. The difference relies on that the airship weight has been reduced to 50 percent (it can mean loss of gas in the blimp or not exact knowledge of its parameters).

Note that the linear velocity errors given in Figure 5(a) have similar time history as for Case 1. The same observation can be made if we consider the angular velocity errors presented in Figure 5(b). It can be concluded that in spite of great reduction of the airship mass the controller still works correctly and gives satisfactory performance. Comparing these errors with the analogous errors for the classical controller, i.e. Figures 5(c) and 5(d), we can observe than the linear velocity errors are only slightly bigger than before. But from Figure 5(d) it results that the angular velocity errors have great values. Hence, we conclude that the classical algorithm is more sensitive for the inertia reduction than the proposed one.

The Figures 6(a) and 6(b) show the forces and torques obtained from the controller. It is noticeable that their the signal values are similar as in Case 1. Comparing the system response with the response in Case 1 it is also observed that the linear velocities are less sensitive for weight reduction than the angular velocity errors. We observe that even by very great weight decreasing realization of the velocity tracking task is possible. Moreover, from Figures $6(\mathrm{c})$ and 6 (d) we see that the control signals have similar values for both controllers.

Taking into account the results of the test it can be concluded that the control algorithm is robust to inertial parameter changes. This means that the algorithm, by taking into account the vehicle dynamics in control gains, can work despite significant changes in the system inertial parameters. 


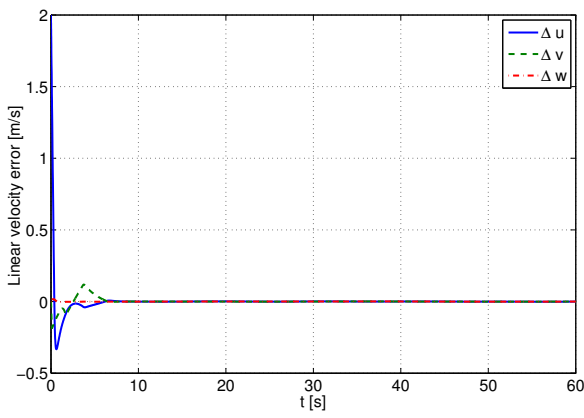

(a)

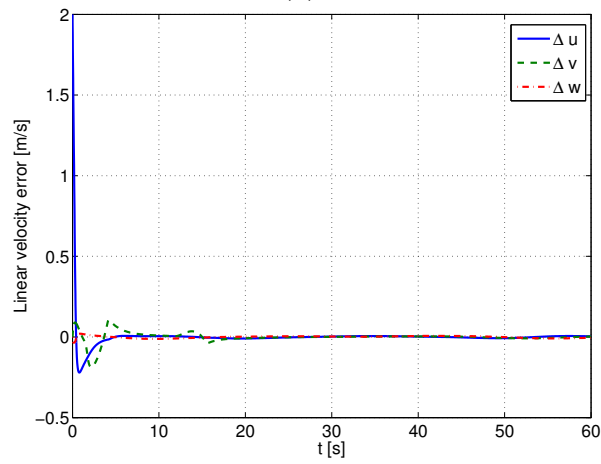

(c)

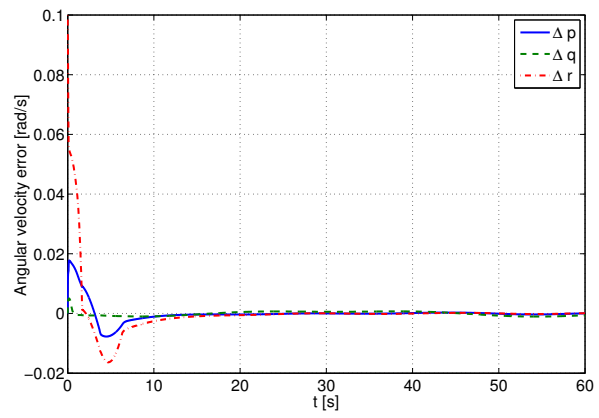

(b)

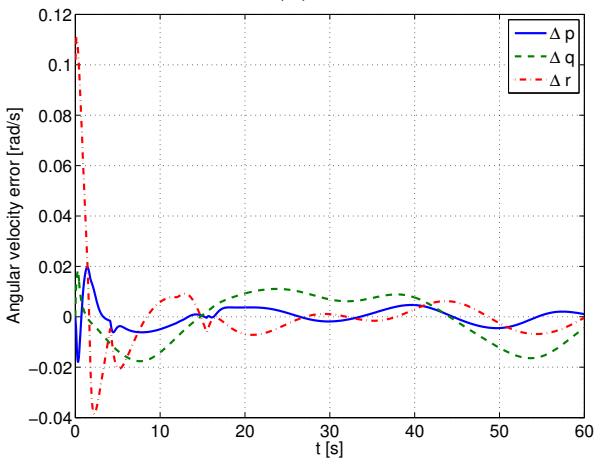

(d)

Figure 5. Case $2-50 \%$ weight reduction $(\Delta \nu \equiv \tilde{\nu})$ : a) (proposed controller) linear velocity errors $\Delta u, \Delta v, \Delta w$, b) (proposed controller) angular velocity errors $\Delta p, \Delta q, \Delta r, \mathrm{c}$ ) (classical controller) linear velocity errors $\Delta u, \Delta v, \Delta w, \mathrm{~d}$ ) (classical controller) angular velocity errors $\Delta p, \Delta q, \Delta r$. 


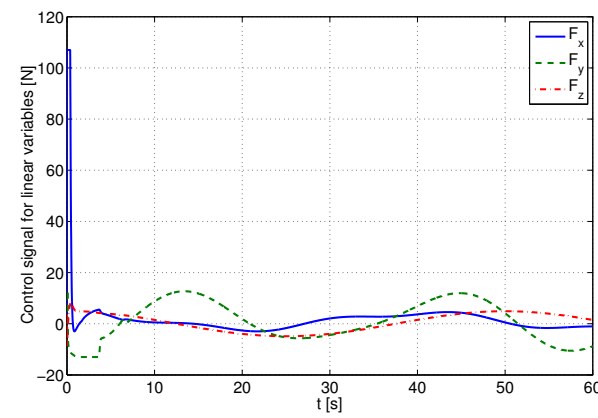

(a)

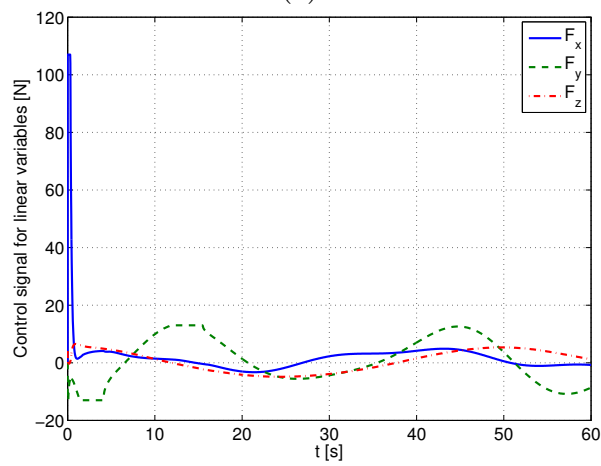

(c)

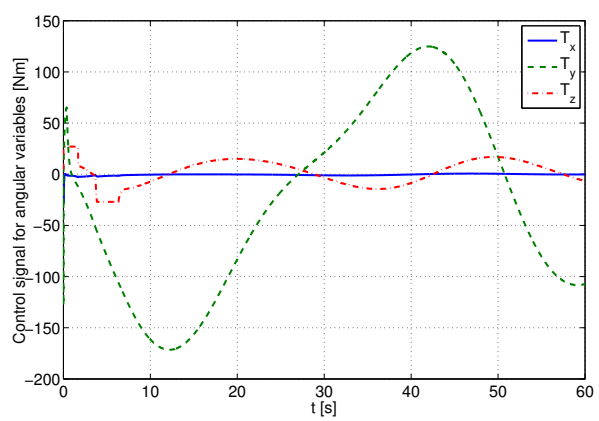

(b)

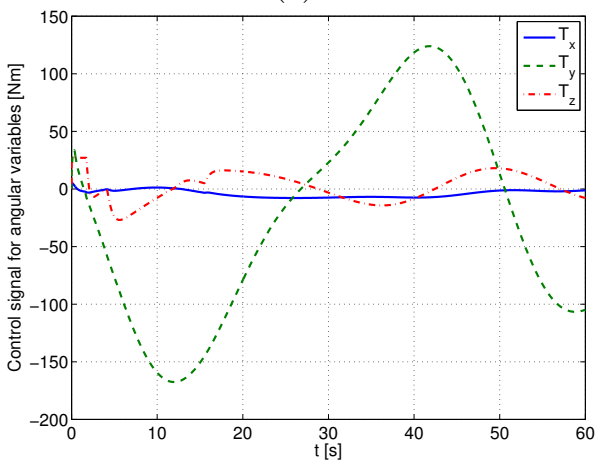

(d)

Figure 6. Case 2 - $50 \%$ weight reduction, control signals: a) (proposed controller) applied forces $F_{x}, F_{y}, F_{z}, \mathrm{~b}$ ) (proposed controller) applied torques $T_{x}, T_{y}, T_{z}, \mathrm{c}$ ) (classical controller) applied forces $F_{x}, F_{y}, F_{z}$, b) (classical controller) applied torques $T_{x}, T_{y}, T_{z}$. 


\section{Conclusions}

In this paper a control algorithm for velocity tracking control is proposed. The nonlinear controller is designed after the velocity transformation. The main property of the algorithm is that the dynamical and geometrical parameters of the vehicle are included in the velocity gain matrix. Consequently, the control coefficients (composed of the control gains and the set of vehicle parameters) are related to the vehicle (they are dependent on its dynamics). The algorithm is universal in the sense that it can be applied for various fully actuated vehicles as marine vehicles, hovercrafts or indoor airships moving with low velocity. Moreover, from its form some simpler controllers can be concluded including the classical form. The formal stability proof was made using Lyapunov direct method. The robustness issue was investigated in the simulation experiment. Simulations for a 6 DOF airship model illustrate the performance of the velocity controller. In the future extension of the controller for underactuated vehicles could be considered.

\section{Acknowledgment}

The work was funded by the National Science Centre of Poland on the basis of the decision DEC-2011/03/B/ST7/02524.

\section{References}

[1] Adamski W., Herman P., Bestaoui Y., Kozlowski K., Control of Airship in Case of Unpredictable Environment Conditions Proceedings of the 2010 Conference on Control and Fault Tolerant Systems, Nice, France, October 6-8, 2010, 843-848.

[2] Ashrafiuon H., Muske K.R., McNinch L.C., Soltan R.A., Sliding-Mode Tracking Control of Surface Vessels IEEE Transactions on Industrial Electronics, 55, 11, 2008, 4004-4012.

[3] Azinheira J.R., Moutinho A., Hover Control of an UAV With Backstepping Design Including Input Saturations IEEE Transactions on Control Systems Technology, 16, 3, 2008, 517-526.

[4] Bestaoui Y., Dynamique dirigeable AS500 complete archive Unpublished report, 2007.

[5] Breivik M., Hovstein V.E., Fossen T.I., Straight-Line Target Tracking for Unmanned Surface Vehicles Modeling, Identification and Control, 29, 4, 2008, 131149 .

[6] Caharija W., Pettersen K.Y., Sorensen A.J., Candeloro M., Gravdahl J.T., Relative velocity control and integral line of sight for path following of autonomous 
surface vessels: Merging intuition with theory Proceedings of IMechE Part M: J Engineering for the Maritime Environment, 228, 2, 2014, 180-191.

[7] Chwa D., Global Tracking Control of Underactuated Ships With Input and Velocity Constraints Using Dynamic Surface Control Method IEEE Transactions on Control Systems Technology, 19, 6, 2011, 1357-1370.

[8] Ferreira B., Matos A., Cruz N., Pinto M., Modeling and Control of the MARES Autonomous Underwater Vehicle Marine Technology Society Journal, 44, 2, 2010, $19-36$.

[9] Fischer N., Hughes D., Walters P., Schwartz E.M., Dixon W.E., Nonlinear RISEBased Control of an Autonomous Underwater Vehicle IEEE Transactions on Robotics, 30, 4, 2014, 845-852.

[10] Fossen T.I., Guidance and Control of Ocean Vehicles John Wiley and Sons, Chichester, 1994.

[11] Fukao T., Fujitani K., Kanade T., Image-based Tracking Control of a Blimp Proceedings of the 42nd IEEE Conference on Decision and Control, Maui, Hawaii USA, December, 2003, 5414-5419.

[12] Garcia-Valdovinos L.G., Salgado-Jimenez T., Bandala-Sanchez M., NavaBalanzar L., Hernandez-Alvarado R., Cruz-Ledesma J.A., Modelling, Design and Robust Control of a Remotely Operated Underwater Vehicle International Journal of Advanced Robotic Systems, 11, 1, 2014, 1-16.

[13] Hayashi R., Osuka K., Ono T., Trajectory Control of an Air Cushion Vehicle Proceedings of the IEEE/RSJ/GI International Conference on Intelligent Robots and Systems '94, Advanced Robotic Systems and the Real World, IROS, 1994, 1906-1913.

[14] Herman P., Normalised-generalised-velocity-component-based controller for a rigid serial manipulator IEE Proceedings - Control Theory \& Applications, 152, 2005, 581-586.

[15] Herman P., About inertial quasi-velocities interpretation and possible application International Journal of Robotics and Automation, 25, 4, 2010, 352-358.

[16] Herman P., Modified set-point controller for underwater vehicles Mathematics and Computers in Simulation, 80, 2010, 2317-2328.

[17] Lapierre L., Soetanto D., Nonlinear path-following control of an AUV Ocean Engineering, 34, 2007, 1734-1744.

[18] Lefeber E., Pettersen K.Y., Nijmeijer H., Tracking control of an underactuated ship IEEE Transactions on Control Systems Technology, 11, 1, 2003, 52-61.

[19] Loduha T.A., Ravani B., On First-Order Decoupling of Equations of Motion for Constrained Dynamical Systems Transactions of the ASME Journal of Applied Mechanics, 62, 1995, 216-222. 
[20] Moutinho A., Mirisola L., Azinheira J., Dias J., Project DIVA: Guidance And Vision Surveillance Techniques for an Autonomous Airship, In: Robotics Research Trends, Xing P. Guo (Ed.) Nova Science Publishers, Inc., New York, 2007, 77-120.

[21] Munoz-Mansilla R., Chaos D., Aranda J., DĂaz J.M., Application of quantitative feedback theory techniques for the control of a non-holonomic underactuated hovercraft IET Control Theory and Applications, 6, 14, 2012, 2188-2197.

[22] Neff A.E., Lee D., Chitrakaran V.K., Dawson D.M., Burg T.C., Velocity Control for a Quad-Rotor UAV Fly-By-Camera Interface Conference Proceedings - IEEE SoutheastCon 2007, Richmond, VA, 22-25 March, 2007, 273-278.

[23] Ohata Y., Ushijima S., Nenchev D.N., Development of an indoor blimp robot with internet-based teleoperation capability Proceedings of the 13th IASTED International Conference on Robotics and Applications, Wurzburg, Germany, August 29-31, 2007, 186-191.

[24] Pota H.R, Ahmed B., Garratt M., Velocity Control of a UAV using Backstepping Control Proceedings of the 45th IEEE Conference on Decision and Control, San Diego, CA, USA, December 13-15, 2006, 5894-5899.

[25] Serres J., Dray D., Ruffier F., Franceschini N., A vision-based autopilot for a miniature air vehicle: joint speed control and lateral obstacle avoidance $A u$ tonomous Robots, 49, 2008, 103-122.

[26] Skjetne R., Fossen T.I., Kokotovic P.V., Adaptive maneuvering, with experiments, for a model ship in a marine control laboratory Automatica, 41, 2005, 289-298.

[27] Slotine J.-J., Li W., Applied Nonlinear Control Prentice Hall, New Jersey, 1991.

[28] Wondergem M., Lefeber E., Pettersen K.Y., Nijmeijer H., Output Feedback Tracking of Ships IEEE Transactions on Control Systems Technology, 19, 2, 2011, 442-448.

[29] Yamasaki T., Goto N., Identification of Blimp Dynamics via Flight Tests Transactions of the Japan Society for Aeronautical and Space Sciences, 46, 153, 2003, 195-205.

[30] Yoon S.-M., Hong S., Park S.-J., Choi J.-S., Kim H.-W., Yeu T.-K., Track velocity control of crawler type underwater mining robot through shallow-water test Journal of Mechanical Science and Technology, 26, 10, 2012, 3291-3298.

[31] Zufferey J.-Ch., Guanella A., Beyeler A., Floreano D., Flying over the reality gap: From simulated to real indoor airships Autonomous Robots, 21, 2006, 243-254.

Received 12.07.2016, Accepted 09.01.2017 